\title{
Theorizing benefits and constraints in collaborative environmental governance: a transdisciplinary social-ecological network approach for empirical investigations
}

\author{
$\underline{\text { Örjan Bodin }}^{1,2}, \underline{\text { Garry Robins }}^{3}, \underline{\text { Ryan R. J. McAllister }}^{4}, \underline{\text { Angela M. Guerrero }}^{5,6}$, Beatrice Crona $^{1,7}$, Maria Tengö $^{1}$ and $^{\text {Mark Lubell }}{ }^{8}$
}

\begin{abstract}
When environmental processes cut across socioeconomic boundaries, traditional top-down government approaches struggle to effectively manage and conserve ecosystems. In such cases, governance arrangements that foster multiactor collaboration are needed. The effectiveness of such arrangements, however, depends on how well any ecological interdependencies across governed ecosystems are aligned with patterns of collaboration. This inherent interdisciplinary and complex problem has impeded progress in developing a better understanding of how to govern ecosystems for conservation in an increasingly interconnected world. We argue for the development of empirically informed theories, which are not only able to transcend disciplinary boundaries, but are also explicit in taking these complex social-ecological interdependences into account. We show how this emerging research frontier can be significantly improved by incorporating recent advances in stochastic modeling of multilevel social networks. An empirical case study from an agricultural landscape in Madagascar is reanalyzed to demonstrate these improvements.
\end{abstract}

Key Words: collaborative governance; connectivity; exponential random graph models (ERGM); interdisciplinary; networks; socialecological fit; social-ecological networks; social-ecological systems

\section{INTRODUCTION}

Ecosystems consist of numerous species and habitats interconnected across geographical and temporal scales (e.g., Christensen et al. 1996). Likewise, human actors ranging from local resource users to actors operating on the global arena are increasingly interconnected through globalized markets, various types of resource flows, and migration (Lambin and Meyfroidt 2011). This social and ecological interconnectedness typically extends beyond single jurisdictions and organizational hierarchies, and global change is likely to further increase the scale and the magnitude of such interconnectivities (Hughes et al. 2013).

Accordingly, a central hypothesis from contemporary research has been that governance arrangements incorporating collaboration across multiple scales and jurisdictions are needed to meet the collective dilemmas arising from a world being socially and ecologically increasingly interconnected (Folke et al. 2005, Klijn and Skelcher 2007, Ansell and Gash 2008, Brondizio et al. 2009, Walker et al. 2009, Cosens 2013, McAllister et al. 2015a).

Building on this general call for collaboration, we argue that it is important to make more precise assertions about how, and under what conditions, collaboration improves governance of complex and boundary-spanning ecosystems. Otherwise, we risk making general recommendations for increasing collaborations among an ever increasing number of local, regional, and global actors; such blanket recommendations are of little value and can even be counterproductive in that large resources might end up being spent on developing collaborative arrangements in cases in which other approaches are more effective. Instead, we argue that effective collaboration requires jointly analyzing both social and ecological connectivity. This requires knowledge of whether, and how, actors interact with each other and which collective action problems these multiactor collaborations are able to address. Simultaneously, effective collaboration requires knowledge on how ecosystems' components interact across various geographical and jurisdictional boundaries.

Different actors are, to varying degrees, constrained by these institutional and jurisdictional boundaries. Thus, the benefits that can be gained from collaboration largely depend on how socioeconomic and ecological structures and processes are aligned to accomplish a good institutional, or social-ecological, fit (Folke et al. 2007, Galaz et al. 2008, Munck af Rosenschöld et al. 2014, Dallimer and Strange 2015, Epstein et al. 2015). Achieving good institutional fit requires, among other things, devising governance structures that are capable of addressing environmental problems at appropriate geographical and functional scales (Cumming et al. 2006, Maciejewski et al. 2015). Hence, to better understand how to accomplish effective collaboration, we need to develop empirically informed theories of institutional fit that explicitly consider complex patterns of social-ecological interdependencies.

Our aim is to address this gap and to help advance theory on effective collaborative arrangements for managing ecosystems spanning different social boundaries. To that end we demonstrate how recent advances in stochastic modeling of multilevel social networks can be integrated with a newly developed socialecological systems (SES) modeling approach, which builds on a network conceptualization (Bodin and Tengö 2012). The

\footnotetext{
${ }^{1}$ Stockholm Resilience Centre, Stockholm University, ${ }^{2}$ Duke University Marine Laboratory, Nicholas School of the Environment, Duke University, ${ }^{3}$ Melbourne School of Psychological Sciences, University of Melbourne, ${ }^{4}$ CSIRO, ${ }^{5}$ Australian Research Council Centre of Excellence for Environmental Decisions, University of Queensland, ${ }^{6}$ School of Biological Sciences, University of Queensland, ${ }^{7}$ Global Economic Dynamics and the Biosphere, Royal Swedish Academy of Sciences, ${ }^{8}$ Department of Environmental Science and Policy, University of California at Davis
} 
stochastic modeling approach extends the class of network models called exponential random graph models (ERGM) developed within the social sciences (e.g., Lusher et al. 2013) and makes it possible to analyze multiple and interconnected networks as one single multilevel network, with each individual network representing one specific level of the multilevel network (Wang et al. 2013, Lazega and Snijders 2015, Lomi et al. 2016). We show how this methodological integration can be applied to theorize about which structures of collaborative relationships might be preferred given specific patterns of ecological interdependencies in managed ecosystems.

Our approach rests on the observation that multilevel networks cannot only represent social networks at different levels, but also the numerous interconnected components of SES. We construe a social-ecological system as networked between two types of nodes at a social and an ecological level (Fig. 1). Social nodes are actors that make resource use decisions and could represent individuals, organizations, or some other social entity. Ecological nodes could include species, ecological communities, or some other ecological concept. Some set of network relationships occur within each set of nodes. For example, actors (social nodes) may be tied through communication or trust, while on the ecological level the movement of animals may connect species communities (ecological nodes) across localities.

Fig. 1. Increasing levels of resolution in our examination of social, ecological, and social-ecological interdependencies (left to right). To the left, all details about social and ecological interdependencies are absent, whereas to the right these interdependencies are described as a social-ecological network. The ties represent actor-to-actor (within the social system), resource-to-resource (within the ecological system), and actorto-resource (across the social and ecological systems) interdependencies.

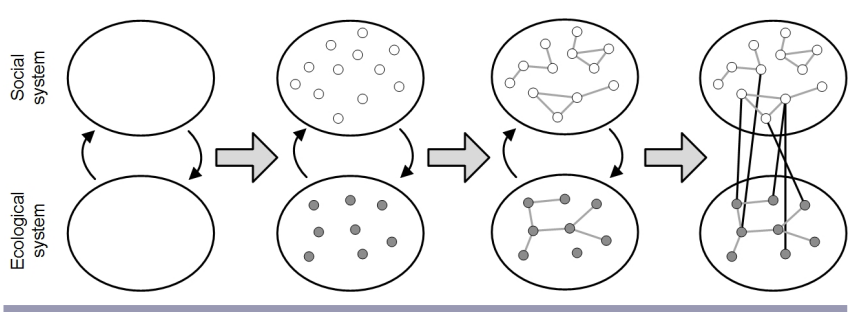

Furthermore, actors typically have different stakes and interests in different components of an ecosystem, which can be conceptualized as cross-level ties going between specific actors and specific ecological resources. Cross-level ties might represent actors using a resource, as when a specific group of fishermen harvest a specific fish stock, or being affected by resource conditions, as when different cities are affected by sea level rise. Different abstractions of social and ecological nodes and ties are feasible, depending on the specific context and the research questions studied (Bodin and Tengö 2012).

We argue that a multilevel social-ecological network perspective is a powerful way to describe and analyze complex patterns of interdependencies in SES. This implies a shift away from models solely based on relationships within a set of state variables, toward a network approach, which emphasizes interdependencies (network ties) between different systems entities (network nodes). Such a shift is congruent with the increased recognition of the complex adaptive systems approach as a means to better understand SESs (Levin 1998, Yletyinen et al. 2016).

Describing an SES as a social-ecological network (as in Fig. 1) does not by itself advance theory of collaborative governance of ecosystems. Theory is needed to identify which structural characteristics of networks might lead to more effective collaborative governance. Recent research has demonstrated how a minimal building block approach allows for a theoretically informed empirical analysis of environmental governance networks (Lubell et al. 2014, McAllister et al. 2014, 2015b, Berardo 2014, Guerrero et al. 2015a), as well as SES expressed as social-ecological networks (Bodin and Tengö 2012, Bodin et al. 2014, Guerrero et al. 2015b). In social-ecological network terms, a social-ecological building block consists of a minimal set of nodes (actors and ecological resources) and ties (their interdependencies) that capture a theoretically important configuration (pattern of social-, ecological-, and socialecological interdependencies). The SES building block approach aims to theoretically link these patterns to specific governance processes and associated governance challenges, and compare the structure of social-ecological networks across cases and contexts. The approach is inherently interdisciplinary because the meaning of a SES building block builds upon an understanding on both social and ecological processes.

Figure 2 further explains this approach by presenting a set of SES building blocks along with a brief description of their associated governance challenges and opportunities (see also Guerrero et al. $2015 b$ for some more examples of governance challenges associated with different SES building blocks). The illustrated building blocks vary according to the presence/absence of specific links. In other words, these configurations capture theoretical assumptions on how certain links between and among actors and resources would be more or less desirable in enabling effective responses to different governance challenges. These SES building blocks only constitute a small subset of all theoretically possible building blocks.

The approach provides a vehicle for the development and empirical testing of theoretically grounded interdisciplinary hypotheses on how different patterns of social-ecological interdependencies relate to collaborative governance outcomes. The combination of theory and method means that not only can we hypothesize particular patterns of effective collaborative structure, but we can then examine these claims against empirical data. For example, if a studied SES is found to be characterized by a strong presence of configurations hypothesized as favorable for effective collaborative environmental governance, one would expect the SES to show evidence of such collaborative effectiveness in terms of, e.g., good ecosystem status and uncoordinated/unregulated resource use and (over)exploitation being kept to a minimum. In this way, we can advance theory on how to accomplish effective collaborative arrangements for environmental governance. 
Fig. 2. A set of minimal social-ecological building blocks and their hypothesized associations with a set of governance challenges. The building blocks capture an assumed relationship between governance processes and particular social-ecological structures. The red nodes represent managing actors, and the green nodes represent ecological resources. The desirable configurations (to the left) differ by the presence of one specific link in relation to the undesirable configurations (to the right). Please observe that these process/structure relationships are only hypothesized, and further research, beyond the scope of this study, is needed to fully test these assumptions.

\begin{tabular}{|c|c|}
\hline $\begin{array}{l}\text { Social-ecological } \\
\text { building block }\end{array}$ & Governance challenge \\
\hline \multicolumn{2}{|c|}{ Common-pool resource management } \\
\hline $\begin{array}{l}\text { Resource sharing with and } \\
\text { without a social tie (closed } \\
\text { and open common pool } \\
\text { triangle) }\end{array}$ & $\begin{array}{l}\text { If two (or more) noncooperating actors share a } \\
\text { resource (right), there may be a strong incentive } \\
\text { for these actors to overharvest the resource. } \\
\text { This governance challenge can, however, be } \\
\text { addressed if the actors collaborate and agree on } \\
\text { some common resource regulations (Ostrom } \\
\text { 1990). This implies that the two actors need to } \\
\text { be socially tied (left). Note that this does not } \\
\text { imply that actors being tied to other actors is } \\
\text { good in general, rather it emphasizes that } \\
\text { collaboration is beneficial for actors sharing } \\
\text { common ecological resources (Bodin et al. } \\
\text { 2014). }\end{array}$ \\
\hline \multicolumn{2}{|c|}{ Social-ecological fit and alignment } \\
\hline $\begin{array}{l}\text { Managing an ecosystem } \\
\text { versus managing a } \\
\text { subcomponent (closed } \\
\text { and open ecosystem } \\
\text { triangles) }\end{array}$ & $\begin{array}{l}\text { If an interconnected ecological resource is } \\
\text { managed as separate entity (right), the } \\
\text { governing structure is not well aligned with the } \\
\text { structure of the ecosystems (Cumming et al. } \\
2006 \text {, Bodin et al. 2014). This governance } \\
\text { challenge resembles the notion of social- } \\
\text { ecological misfit implying that the effect of } \\
\text { management activities can, through ecological } \\
\text { interdependency, spread to other resources } \\
\text { beyond the realm of managing actor. Thus, a } \\
\text { closed triangle (left) hypothetically suggests a } \\
\text { better fitting building block because ecological } \\
\text { costs and benefits occurring beyond the } \\
\text { managed resource are internalized (Bodin et al. } \\
\text { 2014). }\end{array}$ \\
\hline $\begin{array}{l}\text { Two actors managing } \\
\text { interconnected resources } \\
\text { being socially connected } \\
\text { or disconnected (closed } \\
\text { and open four-cycle) }\end{array}$ & $\begin{array}{l}\text { A lack of collaboration between two actors } \\
\text { managing interconnected ecological resources } \\
\text { (right) represents a similar type of governance } \\
\text { challenge as above because the extent of the } \\
\text { interconnected ecological resources is not } \\
\text { aligned with the extent of the governance } \\
\text { structure (social-ecological scale mismatch; } \\
\text { Cumming et al. 2006). If the actors are socially } \\
\text { tied (left), a better social-ecological scale } \\
\text { alignment (fit) is accomplished (Bodin and Tengö } \\
\text { 2012). }\end{array}$ \\
\hline
\end{tabular}

\section{METHODS}

\section{A stochastic multilevel network approach}

A basic approach to studying social-ecological networks is to calculate how frequently different SES building blocks appear in an empirically measured network and compare the observed frequency to the expected frequency from a distribution of networks derived from simple stochastic null models (Bodin and Tengö 2012, Bodin et al. 2014, Kininmonth et al. 2015). This type of frequency analysis is often referred to as conditional uniform random graph test and rests on the assumption of a uniform probability or tie existing between any nodes, although in our case the probability can differ between each node set, i.e., social, ecological, or ecological-social. Theoretical reasoning is used to anchor hypotheses about whether different building blocks are expected to occur with higher or lower frequency relative to the null model, i.e., whether they are enhanced or suppressed. For instance, if there is a tendency for any two actors to collaborate while harvesting the same ecological resource, we expect to see many closed common pool resource (CPR) triangles (Fig. 2) in the social-ecological network. The frequency of such triangles, compared to the frequencies of these triangles in a large number of random networks simulated with the same number of nodes and ties, tells us whether or not the empirically observed network demonstrates a tendency for the actors to collaborate while extracting resources (Ostrom 1990). Thus, the approach implicitly links structure, i.e., the building block, with dynamic socialecological processes, e.g., the tendency for actors sharing a resource to engage in collaboration. The management implications of such collaboration will depend on the specific nature of the collaborative ties being investigated. Hence the precise interpretation will always depend on the theoretical framing and empirical context. However, the approach can begin to elucidate how patterns of collaboration and ecological connectivity together affect governance outcomes.

Using this graph comparison approach to examine the frequencies of different SES building blocks suffers significant empirical limitations (Bodin et al. 2014). For example, because a building block can contain one or several nested instances of another building block, the counts of entangled building blocks are not independent (Milo et al. 2002). To exemplify, the open ecosystem triangle in Figure 2 constitutes a substructure of the open- and closed four-cycles. Furthermore, the frequency counts by themselves are of limited value if there is no baseline measure. Such a baseline measure would rely on devising a large set of random networks. This is a simple enough simulation task, but devising an appropriate stochastic null model is a substantial theoretical challenge (see, e.g., discussion in Borgatti et al. 2009). Such limitations can be mitigated by using multilevel ERGM (Wang et al. 2013).

Exponential random graph models are based on explicit hypotheses about network dependence and build on the idea of analyzing larger networks by studying the presence of smaller configurations, equivalent to what we define as building blocks. These models have their origin in spatial statistics and bear similarity to models in statistical mechanics, but were first introduced as Markov graph models (Frank and Strauss 1986). Extensive development has led to more sophisticated model specifications that can reliably reproduce many of the structural features common in observed social networks (Snijders et al. 2006). As stated, ERGMs have recently been extended to model multilevel network data, i.e., two-layered social networks that are interlinked through cross-level ties (Wang et al. 2013). The term multilevel is deliberately chosen because it conceptually resembles multilevel models, often called hierarchical linear models, although multilevel ERGM is specifically developed for analyzing network data. To emphasize further, even though there are conceptual similarities, the multilevel network models we develop are different from multilevel models defined by the nesting of observations within units, such as people within communities or students within schools (see Raudenbush and Bryk 2002). A 
social-ecological network would, by using this layered network abstraction, be represented as a multilevel network, with the social and the ecological systems at two different levels with network ties representing actor collaboration, ecological interdependencies, and social-ecological interactions, respectively (Fig. 1).

The point of a multilevel ERGM analysis is that, akin to multiple predictors in a regression, multiple building blocks can be considered simultaneously to examine which are most important in explaining the structure of the entire network. Similar to regression analysis, the ERGM gives each of the examined building blocks a parameter estimate and a standard error. The sign of a parameter value is interpreted as whether the associated building block, and the underlying processes giving rise to this building block, are either enhanced or suppressed. The standard error is used to assess statistical significance. So the building blocks whose parameters are not significantly different from zero would be interpreted as neither enhanced nor suppressed.

It should be emphasized, however, that ERGMs are not regressions, most importantly because they take into account the dependencies that are implicit in a network formulation. Regressions assume independent observations, so are not well suited for the analysis of network structure. Exponential random graph models are specified by parameterizing certain graph configurations, equivalent to our building blocks, which imply certain endogenous structural processes in the network (Lusher et al. 2013). They are typically fit by simulation-based estimation procedures (Markov Chain Monte Carlo Maximum Likelihood Estimation - MCMCMLE) that compare simulated graph distributions from initial estimates to the observed network structure and then adjust parameter estimates until the estimates converge.

When fitting a multilevel ERGM to empirical data, the aim is to make empirical inferences about those building blocks required to explain the structure of the network: the frequency of other network patterns can be taken as random noise epiphenomenal to the structure arising from the significant building blocks. Furthermore, attributes of either the social or ecological nodes can be incorporated into the model as explanatory control variables. Nodal attributes can thus be used to distinguish different nodes from each other, e.g., a fishermen from a farmer. Hence, multilevel ERGM is better suited than simple frequency counts to study SES in more complex settings in which assumptions on node similarity are hard to justify. It has also been shown, in other contexts, that nodal attributes at one level may even affect network ties at another (Wang et al. 2014). So, in a social-ecological context, multilevel ERGM has the capacity to examine if features of ecological nodes, for instance, are more likely to encourage collaboration or competition among the actors. Generally, a major benefit of the multilevel approach is the increased ability to take into account network interdependencies across different layers. In sum, the multilevel ERGM then becomes a parsimonious statistical model for the network structure of the entire SES.

\section{An empirical illustration of multilevel exponential random graph model (ERGM)}

In this study, we demonstrate the benefits of multilevel ERGM by drawing on a previous empirical case study in which different clans (social nodes) are managing different forest patches (ecological nodes) in an agricultural landscape in Madagascar (Bodin and Tengö 2012, Bodin et al. 2014). This case study is particularly intriguing because it represents a comparatively successful example of environmental governance. The forest patches have been remarkably well preserved in spite of an increased demand for land and forest resources. Of course, fully testing which building blocks are associated with effective collaboration requires comparison across many more cases. We argue, however, that the case being comparatively successful makes it particularly useful for illustrating the potential of the approach.

The ecological system consists of forest patches that range from 3 to more than 90 ha in size and are scattered across an agricultural landscape of small fields and pastures (Fig. 3). The ecological links represent forest patches interconnected through seed dispersal across the landscape (Bodin et al. 2006). Seed dispersal is a key process for maintaining these forest patches at the landscape level.

Fig. 3. Social-ecological network of forest patches, clans, and their different interrelationships in an agricultural landscape in southern Madagascar. The red nodes represent clans residing in the landscape, and the green nodes forest patches. The tiers between clans represent various forms of social relations, the ties between clans and forest patches represent use and managerial responsibilities, and the ties between the forest patches represent seed dispersals (figure from Bodin and Tengö 2012).

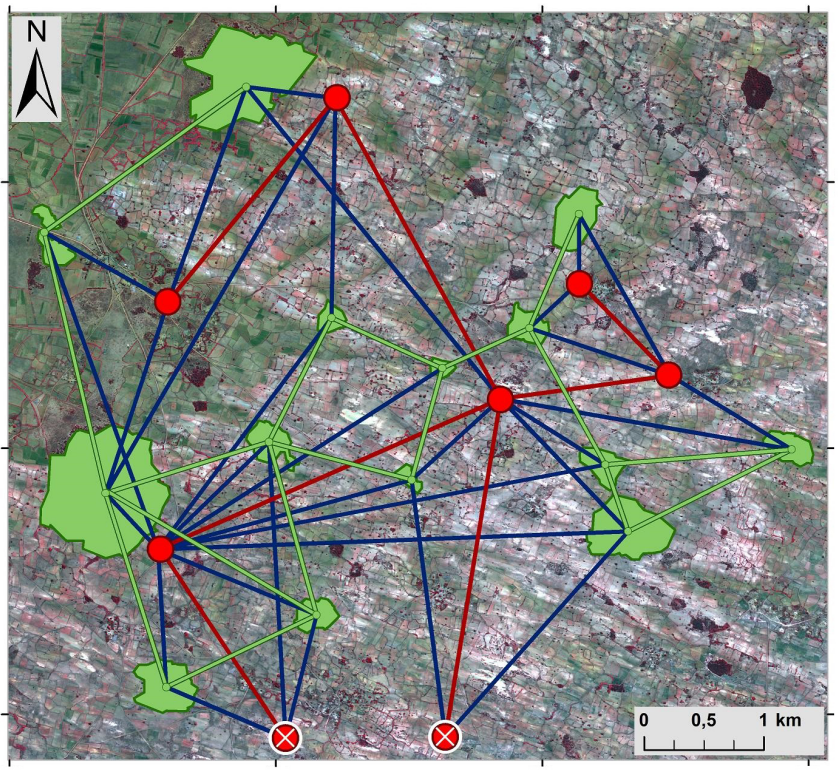

The social links between the clans represent either shared ancestry, agreed kinship that extends over generations, and/or historical dependence relationships between clans. Extensive ethnographic fieldwork and interviews with key informants representing clans have revealed these clan-level relationships, in contrast to links between, e.g., only individuals; all have implications for forest management and access to forest services (Tengö et al. 2007). Hence, by focusing on these clan-level relationships, we only 
considered links that were assessed to be of such strength and nature that they have the potential to affect forest management.

The links between social and ecological nodes are represented by clans that own and/or manage the patches, and regulate use and access through a set of taboo rules. The forest patches have strong cultural importance as sites for burials and other ceremonies, and also generate essential ecosystem services such as microclimate regulation and crop pollination (Tengö et al. 2007). This case is particularly relevant for a social-ecological network analysis because the governance, in effect, involves several interacting actors that all are managing different parts of the landscape, but in which no single actor has authority over the entire SES.

We focused our analysis on the set of six SES building blocks outlined in Figure 2. These explore governance challenges associated with managing common-pool resources and whether and how governance structures are aligned (or not) to the spatial scales of the ecosystems being managed. These building blocks thereby represent hypotheses about structural features of an SES that may be important for environmental governance. Further theoretical and empirical work is needed to more fully explicate major building blocks, so those proposed here should be seen as initial proposals that we investigate in relation to the studied case.

\section{RESULTS}

The results from the frequency analyses (Bodin and Tengö 2012, Bodin et al. 2014) are presented in Figure 4, together with the parameter estimates from the multilevel ERGM. The original Bodin and Tengö (2012) frequency analysis used 28 different statistical tests, whereas the latter analysis used fewer (Bodin et al. 2014). We only used a subsample of all these building blocks. The full ERGM is a simple model with eight parameters, focused around the building blocks of greatest theoretical interest (Fig. 2) and some additional effects that are basic network parameters (to be interpreted as control variables using a multivariate regression analogy, see however Appendix 1 for a more in-depth description of the models and the building blocks). Even though the eight-parameter model is more parsimonious than, for example, the previous 28 variable analyses, post-hoc simulations from the model show that the ERGM reproduces the network data well. This means the underlying processes associated with these eight parameterized building blocks sufficiently explain the observed structure of the entire social-ecological network. The capacity to focus on a parsimonious set of theoretically important building blocks is a major advantage of the ERGM approach.

The results from the two analyses are largely in agreement. Two of the three building blocks associated with favorable socialecological structures (closed CPR triangles and closed ecosystem triangles, see Fig. 2) were found to be enhanced, whereas the third building block (closed four-cycles) was found to be suppressed. Enhanced implies a positive tendency for the actors to configure themselves in accordance with a specific building block, whereas suppressed implies the opposite. The positive parameter estimate (and frequency count) for closed CPR triangles thus gives evidence that clans sharing forest patches tend to collaborate. Also, the positive sign for closed ecosystem triangles demonstrates that clans have a propensity for managing forest patches that are interconnected, which creates tighter feedback loops between the managing activities a clan engages in and the social-ecological effect these activities have on a scale that extends beyond individual forest patches. In other words, it helps to internalize ecological externalities.

Fig. 4. Results from the frequency analysis and the multilevel exponential random graph models (ERGM).

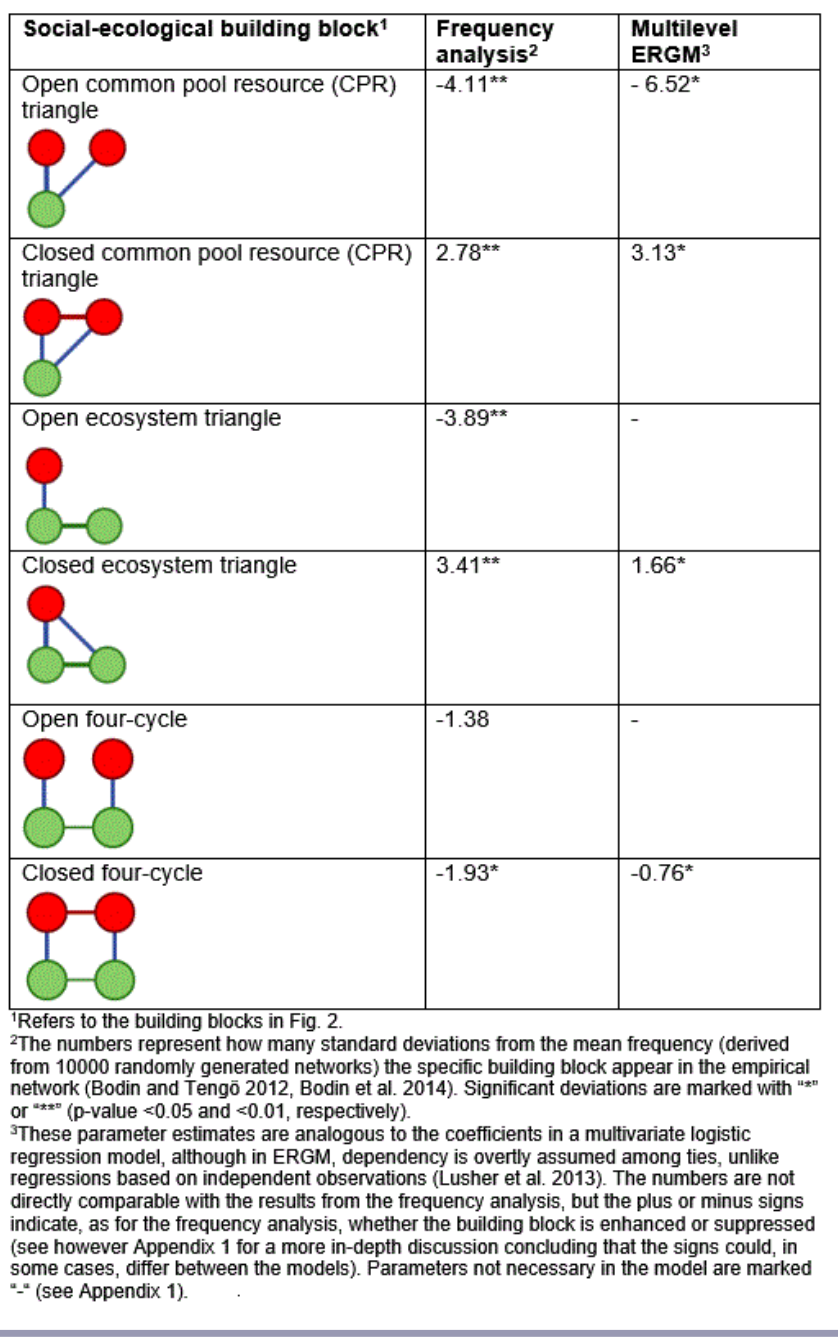

Two building blocks associated with unfavorable social-ecological structures, i.e., open CPR triangles and open ecosystem triangles (Fig. 2), were either suppressed or, in the case of ERGM, not needed to capture the social-ecological network structure. The latter could be interpreted as being neither suppressed nor enhanced (see Appendix 1). These two building blocks can be seen as orthogonal to the presumably favorable building blocks, closed CPR and ecosystem triangles, and the fact that these are suppressed or neutral further strengthens the inferences above. So far, these analyses imply that the social-ecological system is favorably structured to effectively address most of the governance challenges outlined in Figure 2, and thus the results correspond with the observation that the studied case is performing reasonably well (which has been discussed in-depth in previous studies, see, e.g., Bodin and Tengö 2012, Bodin et al. 2014).

Turning to the open ecosystem triangle (Fig. 2), the results from the two analyses differ. The suppression of the hypothesized 
unfavorable open ecosystem triangle was not needed to adequately fit the data using the multilevel ERGM, in contrast to the significant negative effect seen in the frequency analysis (Fig. 4). This deviation illustrates an important difference between the two analyses. To fit these data using multilevel ERGM, we do not need to take into account any (positive or negative) tendency for a clan to manage a patch that is connected to other patches not managed by that clan (open ecosystem triangle). Although there is indeed a tendency for clans to manage pairs (or larger groups) of interconnected patches (i.e., the closed ecosystem triangle, Fig. 2 ), there is, as stated, no tendency either against or for the management of forest patches being ecologically connected to many other patches (open ecosystem triangle). In other words, because of the lack of any such tendency, we expect to see some instances of clans configuring themselves in open ecosystem triangles, even though there is a preference for closed ecosystem triangles. Put differently, if the observed tendency of clans engaging in closed ecosystem triangles was paired with a strong tendency to avoid open ecosystem triangles, we would expect to see no or just a very few instances of the open ecosystem triangle. What the ERGM results therefore imply is that not all ecological interdependencies are under the jurisdiction of single clans (i.e., open ecosystems triangles are expected to be found). This is an important inference about the social processes that underlie the patterns in which clans are given, or take, ownership or control of the different forest patches, and it could not have been arrived at using only the frequency analysis. It also illustrates the value of combining hypotheses of presumingly favorable building blocks with hypotheses on presumingly unfavorable building blocks.

This contrasts with the CPR triangles in which the negative open CPR triangle parameter is significant, and large relative to the other estimates, for both analyses. So, both effects, against open CPR triangles and for closed CPR triangles, are necessary explanations for how the clans configure themselves in managing shared forest patches. Relating this finding with the finding regarding the open and closed ecosystem triangles, it suggests that the clans concentrate more on managing patches that are jointly used (reflected in the CPR triangles) than on management that takes into account the interconnectivity of these patches (open and closed ecosystem triangles).

This same pattern is evident when looking at the more complex four-node building blocks (open and closed four-cycles, Fig. 2). Both ERGM and the frequency analysis show that closed fourcycles are suppressed. Thus, there is a tendency among the clans to avoid collaboration with other clans who are managing forest patches that are interconnected with their own. This, as we argue in Figure 2, is unfavorable when managing interconnected ecological resources, both at this small scale, but also at much larger scales (Hughes et al. 2013, Treml et al. 2015). Essentially, our results suggest that two actors having separate jurisdiction over two ecologically interconnected components tend not to collaborate. This represents an instance of unfavorable socialecological fit (cf. Guerrero et al. 2015b). This partly contradicts our assumption that the relative success of the studied case could be explained by the prevalence of favorable SES building blocks. From the original anthropological data, we know of instances when the absence of collaboration in a closed four-cycle has resulted in management failures and disputes with high social and financial costs (M. Tengö, unpublished data). The relative absence of the closed four-cycles does however not necessarily imply that the SES is doomed to failure. Rather, the absence shows a tendency among clans to steer away from engaging in collaborations that, if realized, presumably could enhance their abilities to manage ecological connectivity between pairs of forest patches (cf. Bergsten et al. 2014).

The building block being orthogonal to the closed four-cycle, i.e., the open four-cycle (Fig. 2), does not have to be included in the ERGM to adequately represent the network (Fig. 4 and Appendix 1). Thus, the open four-cycle should be interpreted as being neither suppressed nor enhanced. Taking this into account, we conclude that the differences between the frequency analysis and ERGM were relatively minor for the open- and closed four-cycle building blocks.

\section{DISCUSSION}

The interdisciplinary social-ecological network modeling framework combined with the recently developed multilevel ERGM approach provides for new and innovative ways of studying social-ecological systems. This can substantially increase our understanding of the processes and structures that make collaborative natural resource governance more or less effective. Our framework complements other research approaches in that it explicitly embraces a complex adaptive systems perspective focusing on patterns of interdependencies between different social and ecological entities. The framework builds on existing studies in which network analysis captures complex interdependences within governance processes (Schneider et al. 2003, Bodin and Crona 2009, Lubell et al. 2014, McAllister et al. 2014, 2015b, Berardo 2014).

A primary advantage of ERGM is that it enables empirical analysis to focus on a limited set of theoretically important building blocks in models that still capture the essential structural characteristics of the entire social-ecological network (see Appendix 1). In contrast, the descriptive frequency analysis often identifies numerous building blocks that deviate from the null model (Bodin and Tengö 2012, Kininmonth et al. 2015), and this complexity, paired with the potential entanglement of the different building blocks, makes it difficult for a researcher to unambiguously identify key underlying social-ecological processes. Multilevel ERGM presents a parsimonious model of how the building blocks (Fig. 2) agglomerate to create the network structure, permitting inferences about whether the structure is consistent with some carefully selected and theoretically informed processes. Although ERGM does not explicitly model the dynamic processes, it nevertheless permits inferences about the structural fingerprints that such processes leave in the network, in the form of structural patterns. It thus provides the researcher with a means to rigorously test hypotheses on the key social-ecological processes that prevail and underpin the observed structures in the studied SES. Methodological work on longitudinal extensions for multilevel ERGMs, to examine how a system may evolve across time, is also under way.

Multilevel ERGMs encourage a research approach starting with simple assumptions on processes or structures, which later can be distilled or extended when the convergence of the ERGM is adequate. That approach, i.e., to start simple and continually refine the model, is commonly applied when using ERGM to study social 
networks (e.g., Robins et al. 2011), and supports the continual refinement of the analysis in an interactive way (see Appendix 1). Furthermore, although we discuss only two-layered networks (one social- and one ecological layer), there is no conceptual constraint on the number of layers that can be investigated simultaneously, although there can be increased computational complexity. In some cases, several layers of both social and ecological networks might be needed to more adequately capture the defining characteristics of the study system.

In conclusion, multilevel ERGM enhances the past approaches to analyzing SES building blocks at the systems level: the issue of constructing a null model is sidestepped; the overlapping nature of different building blocks is dealt with in a statistically rigorous way; various dependencies within and across social and ecological systems can be examined together or separately; and relevant nodal attributes can be incorporated as explanatory factors. Because our study system is characterized by a fairly high degree of homogeneity, the incorporation of nodal attributes was not explicitly considered. In other more complex cases, in which actor and resource heterogeneity is much more prevalent, explicitly taking different nodal attributes into account in the analysis might be necessary.

Considering the increased interest in studying, or advocating, collaboration as a mean to improve governance of natural resources, we argue that multilevel social-ecological network models allow more precise analysis of which actors should be involved in what collaboration, and around what resources, to most effectively address the social-ecological issues at hand. Instead of simplistic assertions that collaboration between all possible pairs of actors is necessarily good, and the more collaborative ties there are, the merrier, the approach can be used to develop explicitly and precisely defined, empirically testable, and theoretically driven hypotheses on how different patterns of interdependencies between and among actors and ecological resources relate to the effectiveness of different collaborative arrangements. With such knowledge, the risk that inefficient, and possibly costly, collaborative relationships are being developed while key collaborative gaps are being ignored could be reduced. Thus, although more research is needed into what relationships between social-ecological structures are important for governance performance (e.g., Fig. 2), this approach holds great promise for progressing the field of sustainability science in an increasingly interconnected and interdependent world.

Although the methodology can be usefully applied to understand singular cases, its strength may lie in the ability to do multicase comparisons in which more generalized conclusions can be drawn regarding which types of SES building blocks, or lack thereof, appear to consistently be associated with specific SES outcomes, and link these to specific SES contexts. Such comparative work would also help highlight inherent trade-offs between SES structures, the types of problems they can address, and the outcomes they can achieve. Social-ecological systems cannot be simultaneously optimized for all types of governance challenges. But, a comparative use of this approach could identify socialecological networks that foster good outcomes across a broad set of possible challenges (cf. Bodin and Crona 2009). The approach supports such a multiobjective perspective by simultaneously examining multiple structural characteristics in a robust and tractable way. Similarly, understanding complex SES benefits from a mixed-methods approach (Young et al. 2006), and the approach presented should be seen as complementary to other tools in the broader theoretical and methodological SES toolbox.

Responses to this article can be read online at: http://www.ecologyandsociety.org/issues/responses. php/8368

\begin{abstract}
Acknowledgments:
$B C, M T$, and $\ddot{O B}$ were supported by MISTRA through a core grant to Stockholm Resilience Centre, Stockholm University. BC was also supported by the Erling-Persson Family Foundation through the Global Economic Dynamics and the Biosphere program at the Royal Swedish Academy of Sciences, Sweden. ÖB acknowledges support from the strategic research program Ekoklim at Stockholm University. $A G$ was supported by the Australian Government's National Environmental Science Program and the Australian Research Council Centre of Excellence for Environmental Decisions.
\end{abstract}

\section{LITERATURE CITED}

Ansell, C., and A. Gash. 2008. Collaborative governance in theory and practice. Journal of Public Administration Research and Theory 18(4):543-571. http://dx.doi.org/10.1093/jopart/mum032

Berardo, R. 2014. Bridging and bonding capital in two-mode collaboration networks. Policy Studies Journal 42(2):197-225. http://dx.doi.org/10.1111/psj.12056

Bergsten, A., D. Galafassi, and Ö. Bodin. 2014. The problem of spatial fit in social-ecological systems: detecting mismatches between ecological connectivity and land management in an urban region. Ecology and Society 19(4):6. http://dx.doi. org/10.5751/es-06931-190406

Bodin, Ö., and B. I. Crona. 2009. The role of social networks in natural resource governance: what relational patterns make a difference? Global Environmental Change 19:366-374. http://dx. doi.org/10.1016/j.gloenvcha.2009.05.002

Bodin, Ö., B. Crona, M. Thyresson, A.-L. Golz, and M. Tengö. 2014. Conservation success as a function of good alignment of social and ecological structures and processes. Conservation Biology 28(5):1371-1379. http://dx. doi.org/10.1111/cobi.12306

Bodin, Ö., and M. Tengö. 2012. Disentangling intangible socialecological systems. Global Environmental Change 22:430-439. http://dx.doi.org/10.1016/j.gloenvcha.2012.01.005

Bodin, Ö., M. Tengö, A. Norman, J. Lundberg, and T. Elmqvist. 2006. The value of small size: loss of forest patches and ecological thresholds in southern Madagascar. Ecological Applications 16 (2):440-451. http://dx.doi.org/10.1890/1051-0761(2006)016[0440: tvossl]2.0.co;2

Borgatti, S. P., A. Mehra, D. J. Brass, and G. Labianca. 2009. Network analysis in the social sciences. Science 323 (5916):892-895. http://dx.doi.org/10.1126/science.1165821 
Brondizio, E. S., E. Ostrom, and O. R. Young. 2009. Connectivity and the governance of multilevel social-ecological systems: the role of social capital. Annual Review of Environment and Resources 34(1):253-278. http://dx.doi.org/10.1146/annurev.environ.020708.100707

Christensen, N. L., A. M. Bartuska, J. H. Brown, S. Carpenter, C. D'Antonio, R. Francis, J. F. Franklin, J. A. MacMahon, R. F. Noss, D. J. Parsons, C. H. Peterson, M. G. Turner, and R. G. Woodmansee. 1996. The report of the Ecological Society of America Committee on the scientific basis for ecosystem management. Ecological Applications 6(3):665-691. http://dx.doi. org/10.2307/2269460

Cosens, B. A. 2013. Legitimacy, adaptation, and resilience in ecosystem management. Ecology and Society 18(1):3. http://dx. doi.org/10.5751/ES-05093-180103

Cumming, G. S., D. H. M. Cumming, and C. L. Redman. 2006. Scale mismatches in social-ecological systems: causes, consequences, and solutions. Ecology and Society 11(1):14. [online] URL: http://www.ecologyandsociety.org/vol11/iss1/ $\underline{\operatorname{art14/}}$

Dallimer, M., and N. Strange. 2015. Why socio-political borders and boundaries matter in conservation. Trends in Ecology and Evolution 30(3):132-139. http://dx.doi.org/10.1016/j.tree.2014.12.004

Epstein, G., J. Pittman, S. M. Alexander, S. Berdej, T. Dyck, U. Kreitmair, K. J. Raithwell, S. Villamayor-Tomas, J. Vogt, and D. Armitage. 2015. Institutional fit and the sustainability of socialecological systems. Current Opinion in Environmental Sustainability 14:34-40. http://dx.doi.org/10.1016/j.cosust.2015.03.005

Folke, C., T. Hahn, P. Olsson, and J. Norberg. 2005. Adaptive governance of social-ecological systems. Annual Review of Environment and Resources 30:441-473. http://dx.doi.org/10.1146/ annurev.energy.30.050504.144511

Folke, C., L. Pritchard, F. Berkes, J. Colding, and U. Svedin. 2007. The problem of fit between ecosystems and institutions: ten years later. Ecology and Society 12(1):30. [online] URL: http://www. ecologyandsociety.org/vol12/iss1/art30/

Frank, O., and D. Strauss. 1986. Markov graphs. Journal of the American Statistical Association 81:832-842. http://dx.doi. org/10.1080/01621459.1986.10478342

Galaz, V., T. Hahn, P. Olsson, C. Folke, and U. Svedin. 2008. The problem of fit among biophysical systems, environmental and resource regimes, and broader governance systems: insights and emerging challenges. Pages 147-186 in O. R. Young, H. Schroeder, and L. A. King, editors. Institutions and environmental change: principal findings, applications, and research frontiers. MIT Press, Cambridge, Massachusetts, USA. http://dx.doi.org/10.7551/ mitpress/9780262240574.003.0005

Guerrero, A. M., Ö. Bodin, R. R. J. McAllister, and K. A. Wilson. 2015b. Achieving social-ecological fit through bottom-up collaborative governance: an empirical investigation. Ecology and Society 20(4):41. http://dx.doi.org/10.5751/ES-08035-200441

Guerrero, A. M., R. R. J. Mcallister, and K. A. Wilson. $2015 a$. Achieving cross-scale collaboration for large scale conservation initiatives. Conservation Letters 8(2):107-117. http://dx.doi. org/10.1111/conl.12112
Hughes, T. P., S. Carpenter, J. Rockström, M. Scheffer, and B. Walker. 2013. Multiscale regime shifts and planetary boundaries. Trends in Ecology and Evolution 28(7):389-395. http://dx.doi. org/10.1016/j.tree.2013.05.019

Kininmonth, S., A. Bergsten, and Ö. Bodin. 2015. Closing the collaborative gap: aligning social and ecological connectivity for better management of interconnected wetlands. AMBIO 44 (S1):138-148. http://dx.doi.org/10.1007/s13280-014-0605-9

Klijn, E.-H., and C. Skelcher. 2007. Democracy and governance networks: compatible or not? Public Administration 85 (3):587-608.

Lambin, E. F., and P. Meyfroidt. 2011. Global land use change, economic globalization, and the looming land scarcity. Proceedings of the National Academy of Sciences 108 (9):3465-3472. http://dx.doi.org/10.1073/pnas.1100480108

Lazega, E., and T. A. B. Snijders, editors. 2015. Multilevel network analysis for the social sciences: theory, methods and applications. Springer, Berlin, Germany. http://dx.doi.org/10.1007/978-3-319-24520-1

Levin, S. A. 1998. Ecosystems and the biosphere as complex adaptive systems. Ecosystems 1(5):431-436. http://dx.doi. org/10.1007/s100219900037

Lomi, A., G. Robins, and M. Tranmer. 2016. Introduction to multilevel social networks. Social Networks 44:266-268. http://dx. doi.org/10.1016/j.socnet.2015.10.006

Lubell, M., G. Robins, and P. Wang. 2014. Network structure and institutional complexity in an ecology of water management games. Ecology and Society 19(4):23. http://dx.doi.org/10.5751/ es-06880-190423

Lusher, D., J. Koskinen, and G. Robins. 2013. Exponential random graph models for social networks: theory, methods, and applications. Cambridge University Press, Cambridge, Massachusetts, USA. http://dx.doi.org/10.1017/cbo9780511894701

Maciejewski, K., A. De Vos, G. S. Cumming, C. Moore, and D. Biggs. 2015. Cross-scale feedbacks and scale mismatches as influences on cultural services and the resilience of protected areas. Ecological Applications 25(1):11-23. http://dx.doi. org/10.1890/13-2240.1

McAllister, R. R. J., R. McCrea, and M. N. Lubell. 2014. Policy networks, stakeholder interactions and climate adaptation in the region of South East Queensland, Australia. Regional Environmental Change 14(2):527-539. http://dx.doi.org/10.1007/ s10113-013-0489-4

McAllister, R. R. J., C. J. Robinson, K. Maclean, A. M. Guerrero, K. Collins, B. M. Taylor, and P. J. De Barro. 2015a. From local to central: a network analysis of who manages plant pest and disease outbreaks across scales. Ecology and Society 20(1):67. http://dx.doi.org/10.5751/ES-07469-200167

McAllister, R. R. J., B. M. Taylor, and B. P. Harman. $2015 b$. Partnership networks for urban development: how structure is shaped by risk. Policy Studies Journal 43(3):379-398. http://dx. doi.org/10.1111/psj.12103

Milo, R., S. Shen-Orr, S. Itzkovitz, N. Kashtan, D. Chklovskii, and U. Alon. 2002. Network motifs: simple building blocks of 
complex networks. Science 298(5594):824-827. http://dx.doi. org/10.1126/science.298.5594.824

Munck af Rosenschöld, J., N. Honkela, and J. I. Hukkinen. 2014. Addressing the temporal fit of institutions: the regulation of endocrine-disrupting chemicals in Europe. Ecology and Society 19(4):30. http://dx.doi.org/10.5751/es-07033-190430

Ostrom, E. 1990. Governing the commons: the evolution of institutions for collective action. Cambridge University Press, Cambridge, Massachusetts, USA.

Raudenbush, S. W., and A. S. Bryk. 2002. Hierarchical linear models: applications and data analysis methods. Sage, Thousand Oaks, California, USA.

Robins, G., L. Bates, and P. Pattison. 2011. Network governance and environmental management: conflict and cooperation. Public Administration 89(4):1293-1313. http://dx.doi.org/10.1111/ j.1467-9299.2010.01884.x

Schneider, M., J. Scholz, M. Lubell, D. Mindruta, and M. Edwardsen. 2003. Building consensual institutions: networks and the National Estuary Program. American Journal of Political Science 47(1):143-158. http://dx.doi.org/10.1111/1540-5907.00010

Snijders, T. A. B., P. E. Pattison, G. L. Robins, and M. S. Handcock. 2006. New specifications for exponential random graph models. Sociological Methodology 36(1):99-153. http://dx. doi.org/10.1111/j.1467-9531.2006.00176.x

Tengö, M., K. Johansson, F. Rakotondrasoa, J. Lundberg, J.-A. Andriamaherilala, J.-A. Rakotoarisoa, and T. Elmqvist. 2007. Taboos and forest governance: informal protection of hot spot dry forest in southern Madagascar. AMBIO 36(8):683-691. http:// dx.doi.org/10.1579/0044-7447(2007)36[683:tafgip]2.0.co;2

Treml, E., P. I. J. Fidelman, S. Kininmonth, J. A. Ekstrom, and Ö. Bodin. 2015. Analyzing the (mis)fit between the institutional and ecological networks of the Indo-West Pacific. Global Environmental Change 31:263-271. http://dx.doi.org/10.1016/j. gloenvcha.2015.01.012

Walker, B., S. Barrett, S. Polasky, V. Galaz, C. Folke, G. Engström, F. Ackerman, K. Arrow, S. Carpenter, K. Chopra, G. Daily, P. Ehrlich, T. Hughes, N. Kautsky, S. Levin, K.-G. Mäler, J. Shogren, J. Vincent, T. Xepapadeas, and A. de Zeeuw. 2009. Looming global-scale failures and missing institutions. Science 325 (5946):1345-1346. http://dx.doi.org/10.1126/science.1175325

Wang, P., G. Robins, P. Pattison, and E. Lazega. 2013. Exponential random graph models for multilevel networks. Social Networks 35(1):96-115. http://dx.doi.org/10.1016/j.socnet.2013.01.004

Yletyinen, J., Ö. Bodin, B. Weigel, M. C. Nordström, E. Bonsdorff, and T. Blenckner. 2016. Regime shifts in marine communities: a complex systems perspective on food web dynamics. Proceedings of the Royal Society B: Biological Sciences 283(1825):20152569. http://dx.doi.org/10.1098/rspb.2015.2569
Young, O. R., E. F. Lambin, F. Alcock, H. Haberl, S. I. Karlsson, W. J. McConnell, T. Myint, C. Pahl-Wostl, C. Polsky, P. S. Ramakrishnan, H. Schroeder, M. Scouvart, and P. H. Verburg. 2006. A portfolio approach to analyzing complex humanenvironment interactions: institutions and land change. Ecology and Society 11(2):31. [online] URL: http://www.ecologyandsociety. org/vol11/iss $2 / \operatorname{art31/}$ 


\section{Appendix 1.}

Fitting a multilevel ERGM

First, the structure of the ecological network was considered exogenous and not liable to change. Thus it was held constant (fixed) in estimating the ERGM. Secondly, no triangles were observed in the social network, so we controlled the estimation so that social triangles were impossible. We then started with a simple model where we only included the favorable building blocks (Main paper, Fig. 2) and edge configurations for both network layers (to control for the density of links in the social network and the cross-level network). The results are presented in Fig. A1.1. We here use the naming convention of configurations (building blocks) as outlined in recent studies (Wang et al. 2013)

Figure A1.1. A fitted ERGM with a minimal number of tested building blocks. We use the terminology for building blocks commonly used in the ERGM literature. The social network is denoted " $A$ ", the ecological network " $B$ ", and the cross-level ties as " $X$ ". Significant estimates are marked with *.

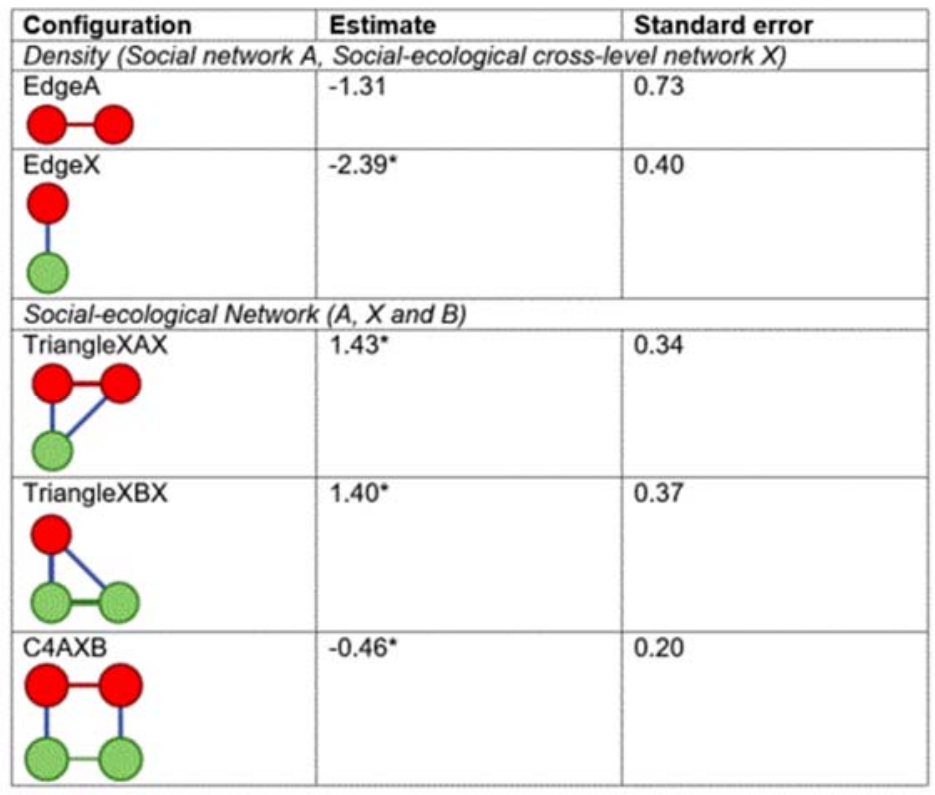

This first model was then extended to account for centralization, i.e. that some nodes in the social and ecological network are more connected than others (Fig. A1.2). These parameters capture an important network feature, controlling for degree distributions. Arguably, network models should always include degree distribution parameters, given the relevance of degree-based effects (Barabási and Albert 1999), and so model 1 should be treated as a simplified version that focusses only on a few theoretically relevant building blocks. 
Figure A1.2. A fitted ERGM (from Fig. A1.1) including centralization parameters (prefixed Star2).

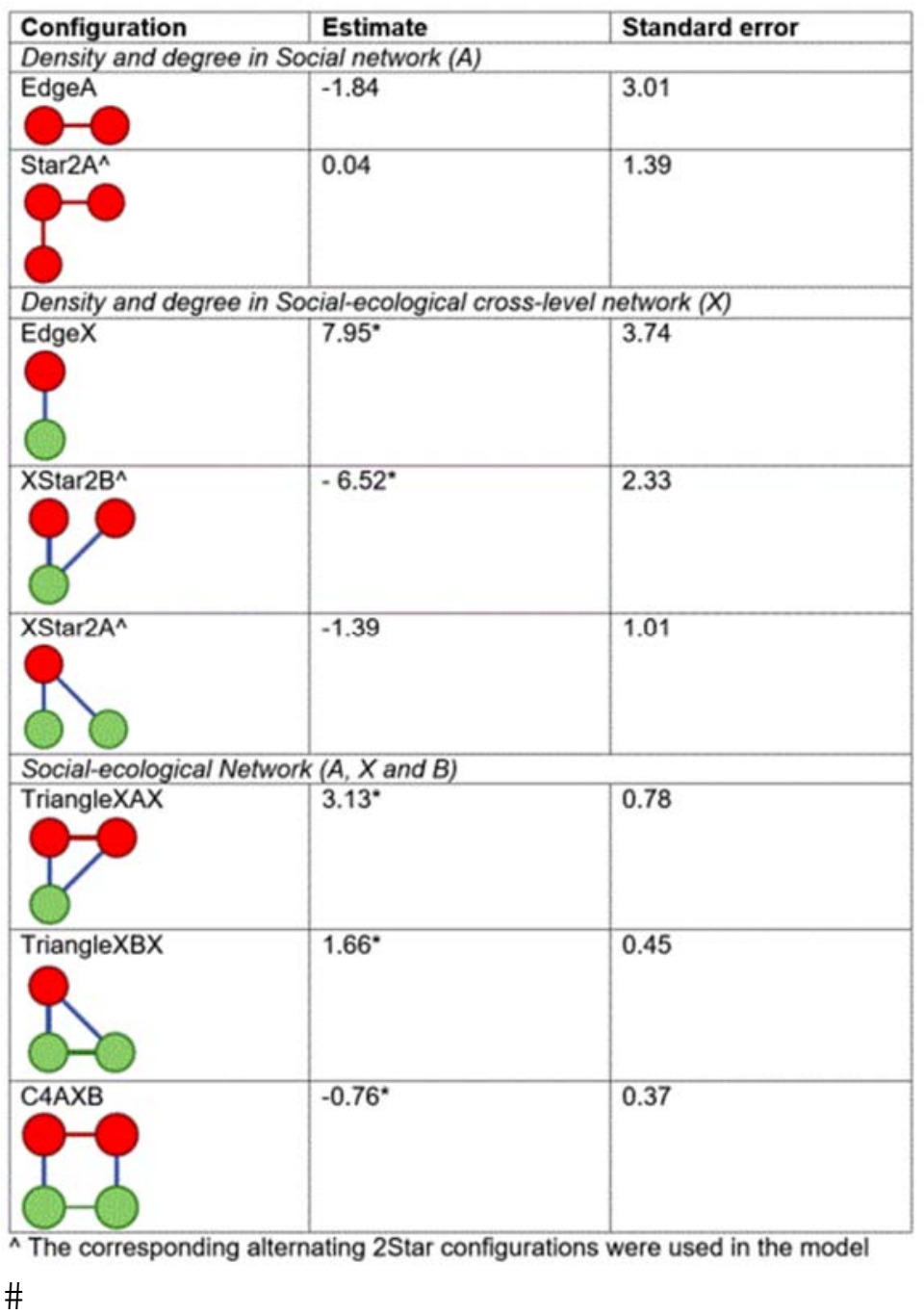

The centralization parameters are prefixed "Star2" in Fig. A1.2. Given the multilevel network structure, there are three degree distributions of interest:

- Centralization among ties in the clan network only (Star2A -Are there highly central clans in the social network?)

- Centralization of clans in social-ecological ties (XStar2A - Are there clans that are highly central in ties to forests?)

- Centralization of forests in social-ecological ties (XStar2B - Are there forests that are highly central in links to clans?)

A fourth possible degree distribution, among ecological ties, cannot be parameterized because as explained above, the ecological network is treated as exogenous.

The centralization parameters are all based on the alternating-star parameters (configurations) for ERGMs as explained in Lusher et al (2013). Without going 
into the details here, the strongest effect in the alternating star parameter is a so-called 2Star which is a network path of length two centered on one node (hence its contribution to a centralization parameter - higher order stars are also included in the statistic as set out in p.66 of Lusher et al. 2013). So, the alternating XStar2B configuration can also be interpreted as deriving from the open common pool resource triangle in Fig. 2 (main paper).

In contrast, the configuration XStar2A derives from a configuration centered on a clan connected to two forests.

Note that the inclusion of the centralization parameters substantially changes the estimate of the TriangleXAX. The XStar2B, included in the alternating $\mathrm{XStar2B}$ configuration, is a lower order configuration to the triangle (i.e. TriangleXAX contains the XStar2B centered on the forest in the triangle). As explained in (Snijders et al. 2006), the inclusion of lower order effects in triangulation sharpens the inference about the formation of triangles. With the centralization parameters and the triangle parameter present, the model in effect asks the question: given the presence of an XStar2B, what is the likelihood of a tie between the two social nodes? Or to put it more substantively: given that two clans manage the same forest, what is the likelihood that they will be socially linked? This is a sharper inference than in model 1 (Fig. A1.1) which simply asks about the presence of the triangle in the graph conditional on the density (captured by the edge parameters) and the other cross-level effects.

Although this conditionality in interpretation is often downplayed in ERGMs to simplify the detail (as we have largely done in the main paper), it is an appealing feature of the statistical model when a more fine grained inference about possible processes is required (For a further discussion of inference about likely structural processes from these models, see Lusher et al, 2013, chapter 3). This also represent an important distinction between ERGM and the simpler frequency counting approach. There could very well be cases where lower order effects are strongly positive, whereas higher order effects are weakly negative. In such cases, the frequency of higher order configuration could deviate positively from the expected mean given a random network, but this deviance would be to the result of the strong positive effect of the lower order configuration that overshadows the weaker and negative effect of the higher order configuration.

These issues should not be confused with technical issues such as multicollinearity in regression. The Markov Chain Monte Carlo Maximum Likelihood Estimation (MCMCMLE) used to estimate these models can successfully pick apart highly correlated effects. If there is too much collinearity, the models will not converge. The post-estimation goodness of fit simulation described below can reassure that the models are behaving properly and that the estimates are indeed maximum likelihood in producing distributions with all parameterized network statistics centered on the observed values. 
Similarly, the increase in the standard error for TriangleXAX across models reflects the focus on a smaller number of "observations" (i.e. where XStar2B are present).

Conclusively, the TriangleXAX parameter in Model 2 (Fig. A1.2) concentrates the inference on the formation of the social tie in the social-ecological configuration, rather than just the presence of the configuration overall (model 1). Comparison of the estimate in model 2 with the smaller value in model 1 suggests that the tendency for two clans managing the same forest to have a social tie is a strong effect. It is stronger than the effect for just the presence of the triangle "on average" (i.e. in model 1).

The overall fit improved with these additions, as measured by the Mahalanobis distance that decreased from 803 to 263 (the distance captures the level of fit, see e.g Lusher et al. 2013). Note that no other building blocks were included in the model. We tested to include the remaining building blocks in Fig. 2 (main paper) by simulating from the model estimates (the socalled goodness of fit test - see Lusher et al, 2013). A post-estimation simulation can confirm that the model has successfully converged, that the statistics from the fitted parameters are indeed central in the distributions of statistics derived from the graph distribution (i.e. they are maximum likelihood), and that relevant non-fitted observed graph statistics (configurations) are not extreme compared to the distribution of statistics derived from the simulation (i.e., the model is not inconsistent with these additional structural effects).

None of the observed counts of non-fitted building blocks were extreme in the distribution of graph statistics produced from the simulations. The established index of whether a count is extreme is the t-statistic, with a value of less than two in absolute value for non-fitted effects suggesting that the model is plausible in explaining that effect (for fitted effects, it is desirable to have a value less than around 0.2 to confirm convergence - see Lusher et al, 2013.) For the six building blocks in Figure 2, the t-statistics for a simulated sample of 1000 graphs were:

- Fitted effects

o Closed CPR triangle: -0.01

o Closed Ecosystem triangle: 0.04

o Closed four cycle: 0.02

o Open CPR triangle: -0.12 (alternating XStar2B was fitted)

- Effects not directly fitted

o Open Ecosystem triangle: -0.3

o Open four cycle: 0.47

Thus we conclude that the non-fitted effects (i.e. the SE building blocks open four cycle and open ecosystem triangle in Fig. 2, main paper) are not necessary to explain the network structure over and above the given model parameters. This implies that the open ecosystem triangle and the open fourcycle were neither suppressed nor enhanced. 


\section{Literature Cited}

Barabási, A. L., and R. Albert. 1999. Emergence of scaling in random networks. Science 286:509-512.

Lusher, D., J. Koskinen, and G. Robins. 2013. Exponential Random Graph Models for Social Networks: Theory, Methods, and Applications. Cambridge University Press, Cambridge.

Snijders, T. A. B., P. E. Pattison, G. L. Robins, and M. S. Handcock. 2006. New specifications for exponential random graph models. Sociological Methodology 36(1):99-153.

Wang, P., G. Robins, P. Pattison, and E. Lazega. 2013. Exponential random graph models for multilevel networks. Social Networks 35(1):96-115. 